\title{
A Ranking Method Based on Extended Possibility Degree Dominance Relation
}

\author{
Qiwen Zhang ${ }^{1}$ and Xueqin Wang ${ }^{1}$ \\ ${ }^{1}$ School of Computer and Communication, Lanzhou University of Technology, \\ Lanzhou 730050, China \\ qingwenzhang@lut.cnwxueqin888@163.com
}

\begin{abstract}
Dominance relation is an important method in incomplete interval-valued information systems. In order to solve the problem that too many attributes maybe result in the ranking failure in the incomplete interval-valued information systems, the concepts of extended possibility degree, extended possibility degree dominance relation and average comprehensive dominance degree are defined. Based on them, a ranking method based on extended possibility degree dominance relation is proposed. Finally, comparing with other ranking methods, this new ranking method based on extended possibility degree dominance relation not only has stronger differentiation degree, but also can make ranking results more objective and reasonable.
\end{abstract}

Keywords: incomplete interval-value, possibility degree, average comprehensive dominance degree, ranking, differentiation degree

\section{Introduction}

Rough set is a theory of mathematical analysis proposed by a Polish scholar Pawlak[1] in 1982. Classical rough set theory is established based on equivalence relation and applied to the complete systems. Since then, Greco et al proposed dominance-based rough sets approach (DRSA) [2-4]. But in reality life, a lot of information is incomplete and exists in the form of interval-value mostly. The ranking is an important problem in the incomplete interval-valued information systems. How to find a high differentiation degree, quick, simple, reasonable and effective way to distinguish the decision-making objects is an important research.

Subsequently, ranking researches for decision-making objects based on dominance relation are emerging. Liu et al. studied the ranking research based on dominance relation for multiple-attribute $[5,6]$. Chen et al. proposed the concept of tolerance dominance relation for dealing with the ranking failure problem caused by the too strict classic dominance relation [7]. Wang et al. created a comprehensive ranking method based on the combination of dominance relation and entropy weight [8]. Li et al. created a ranking method based on dominance relation to solve the incomplete multiple-attribute decision-making problem [9]. Zhao et al. proposed a new way to fill the incomplete information system for ranking [10]. Qiu et al. proposed a ranking method about interval numbers based on probability reliability [11]. Yang et al. proposed a $\alpha$ dominance relation [12]. Hu et al. proposed a new $\alpha-\beta$ dominance relation to solve multiple-attribute decision-making problems about interval numbers [13]. Wang et al. proposed a ceiling dominance relation and approximate dominance relation for the problems that are existing in the incomplete interval-valued information systems [14]. Yang et al. proposed an extension probability dominance relation [15]. 
Based on above researches, in order to solve the problem that too many attributes maybe result in the ranking failure in the incomplete interval-valued information systems, a ranking method based on extended possibility degree dominance relation is proposed. This new ranking method can not only get rules from incomplete information systems directly, but also make the ranking result have higher differentiation degree. Besides, it can make the ranking result more objective and reasonable.

\section{Incomplete Interval-valued Information System}

Definition 1: An interval-value information system is a quadruple $S=(U, A, V, f)$, where $U=\left\{x_{1}, x_{2} \cdot \cdot, x_{n}\right\}$ is a finite non-empty set of objects and $A=\left\{a_{1}, a_{2}, \cdots, a_{m}\right\}$ is a finite non-empty set of attributes, $V=\bigcup_{a \in A} V_{a}$ is a set of domains of attributes, and then $V_{a}$ is an interval-value and represents the range of the attribute $a$ value. Denoted by $f(x, a)=\left[a^{L}(x), a^{U}(x)\right]$, where $a^{L}(x), a^{U}(x) \in R$ and $a^{L}(x) \leq a^{U}(x), a^{L}(x)$ is the left endpoint of $f(x, a), a^{U}(x)$ is the right endpoint of $f(x, a), S$ is called an intervalvalue information system. In particular, if $a^{L}(x)=a^{U}(x)$, then $f(x, a)$ would degenerate into a real number. Given $V=\bigcup_{a \in A} V_{a} \bigcup_{\{*\}}$, where * expresses unknown attribute values, denoted by $f(x, a)=\left[a^{L}(x),{ }^{*}\right], f(x, a)=\left[*, a^{U}(x)\right]$ and $f(x, a)=\left[*^{L}, *^{U}\right]$, $S$ is called an incomplete interval-valued information system.

\section{Improved Possibility Degree Dominance Relation}

\subsection{Extension Possibility Degree in the Incomplete Interval-Valued Information System}

At present, there are two ways to deal with the incomplete interval-valued information system. One is transforming the incomplete information into the complete information for ranking, and the other is getting rules from the incomplete interval-valued information system for ranking.

(1) Wang et al proposed two ranking methods. One is that the incomplete information is filled firstly and the ceiling dominance relation is used for ranking secondly, the other is that the approximate dominance relation is used for ranking [14].

The concrete way that fills incomplete information is following:

1) if $f(x, a)=\left[a^{L}(x),{ }^{*}\right]$, then $*=\max \left\{a^{L}(x), \max _{x \in U}\left\{a^{U}(x)\right\}\right\}$;

2) if $f(x, a)=\left[*, a^{U}(x)\right]$, then $*=\min \left\{a^{U}(x), \min _{x \in U}\left\{a^{L}(x)\right\}\right\}$;

3) if $f(x, a)=\left[*^{L}, *^{U}\right]$, then $*^{L}=\min _{x \in U}\left\{a^{L}(x)\right\}, *^{U}=\max _{x \in U}\left\{a^{U}(x)\right\}$.

The ceiling dominance relation $R_{B}^{2}$ and its class of advantage $\left[x_{i}\right]_{B}^{\geq}$about $B$ are defined as:

$$
\begin{gathered}
R_{B}^{\geq}=\left\{\left(x_{j}, x_{i}\right) \in U \times U \mid a^{U}\left(x_{j}\right) \geq a^{U}\left(x_{i}\right), \forall \mathrm{a} \in B\right\} \\
{\left[x_{i}\right]_{B}^{\geq}=\left\{x_{j} \in U \mid\left(x_{j}, x_{i}\right) \in \mathrm{R}_{B}^{\geq}\right\}}
\end{gathered}
$$

The approximate dominance relation $R_{B}^{\geq}$and its class of advantage $\left[x_{i}\right]_{B}^{2}$ about $B$ are defined as:

$$
\begin{gathered}
R_{B}^{\geq}=\left\{\left(x_{j}, x_{i}\right) \in U \times U \mid a^{U}\left(x_{j}\right) \geq a^{U}\left(x_{i}\right) \vee a^{U}\left(x_{i}\right)=* \vee a^{U}\left(x_{j}\right)=*, \forall \mathrm{a} \in B\right\} \\
{\left[x_{i}\right]_{B}^{\geq}=\left\{x_{j} \in U \mid\left(x_{j}, x_{i}\right) \in \mathrm{R}_{B}^{\geq}\right\}}
\end{gathered}
$$


(2) Yang [15] proposed extended probability dominance relation $R_{B}^{2}$ and its class of advantage $\left[x_{i}\right]_{B}^{\geq}$about $B$ are defined as:

$$
R_{B}^{\geq}=\left\{\left(x_{j}, x_{i}\right) \in U \times U \mid \partial_{j i}^{a} \geq 0.5 \vee\left(\partial_{j i}^{a}=0.5 \wedge\left|f\left(x_{i}, a\right)\right| \geq\left|f\left(x_{j}, a\right)\right|\right) \vee\left(\partial_{j i}^{a}=*\right), \forall a \in B\right\}
$$

if $f\left(x_{i}, a\right)=* \vee f\left(x_{j}, a\right)=*$, then $\partial_{j i}^{a}=*$.

$$
\left[x_{i}\right]_{B}^{2}=\left\{x_{j} \in U \mid\left(x_{j}, x_{i}\right) \in \mathrm{R}_{B}^{\geq}\right\}
$$

Above methods are defective after analysis. The specific defects are as follows:

(1) The filling method will bring about the deviation between the new data and the original data. Moreover, the ceiling dominance relation is too loose. For example, according to the definition of ceiling dominance relation, $[1,100.01]$ is superior to $[99,100]$, but in fact, the possibility that $[99,100]$ is superior to $[1,100.01]$ is bigger. Therefore, error classification will cause unreasonable ranking result. Besides, the definition of approximate dominance relation is also too loose. For example, according to the definition of approximate dominance relation, [3,5] is superior to $\left[6,{ }^{*}\right]$, but in fact, [3,5] will never be superior to $[6, *]$ for any *. Therefore, error classification will cause unreasonable ranking result, too.

(2) The definition of extended probability dominance relation is too strict. For example, according to the definition of extended probability dominance relation, $[4,9]$ and $[3, *]$ will not to be compared; $[6,7]$ is superior to $[4,9]$. But in fact, the probabilities of mutual advantages of their attributes in two interval are equal and this does not mean that $[6,7]$ must be superior to $[4,9]$.

Though above analysis, this paper defines extended possibility degree based on the possibility degree.

Definition 2: Given an incomplete interval-value information system $S=(U, A, V, f)$, extended possibility degree under the attribute $a$ is defined as:

If $f\left(x_{i}, a\right)=\left[a^{L}\left(x_{i}\right), a^{U}\left(x_{i}\right)\right], f\left(x_{j}, a\right)=\left[a^{L}\left(x_{j}\right), a^{U}\left(x_{j}\right)\right.$, then

$$
\partial_{j i}^{a}=\frac{\min \left\{l_{i}^{a}+l_{j}^{a}, \max \left\{\mathrm{a}^{U}\left(x_{j}\right)-\mathrm{a}^{L}\left(x_{i}\right), 0\right\}\right\}}{l_{i}^{a}+l_{j}^{a}}
$$

where, $l_{i}^{a}=a^{U}\left(x_{i}\right)-a^{L}\left(x_{i}\right), l_{j}^{a}=a^{U}\left(x_{j}\right)-a^{L}\left(x_{j}\right)$.

If $f\left(x_{i}, a\right)=\left[a^{L}\left(x_{i}\right),{ }^{*}\right], f\left(x_{j}, a\right)=\left[a^{L}\left(x_{j}\right), a^{U}\left(x_{j}\right)\right.$. or $f\left(x_{i}, a\right)=\left[a^{L}\left(x_{i}\right), a^{U}\left(x_{i}\right)\right], \quad f\left(x_{j}, a\right)=\left[{ }^{*}, a^{U}\left(x_{j}\right)\right]$ or $f\left(x_{i}, a\right)=\left[a^{L}\left(x_{i}\right){ }^{*}\right], f\left(x_{j}, a\right)=\left[*, a^{U}\left(x_{j}\right)\right]$ and $a^{U}\left(x_{j}\right) \leq a^{L}\left(x_{i}\right)$, then $\partial^{* a}{ }_{j i}=0$.

If $f\left(x_{i}, a\right)=\left[a^{L}\left(x_{i}\right), a^{U}\left(x_{i}\right)\right], f\left(x_{j}, a\right)=\left[a^{L}\left(x_{j}\right), *\right]$ or $f\left(x_{i}, a\right)=\left[{ }^{*}, a^{U}\left(x_{i}\right)\right], f\left(x_{j}, a\right)=\left[a^{L}\left(x_{j}\right), a^{U}\left(x_{j}\right)\right]$ or $f\left(x_{i}, a\right)=\left[{ }^{*}, a^{U}\left(x_{i}\right)\right], f\left(x_{j}, a\right)=\left[a^{L}\left(x_{j}\right), *\right]$ and $a^{U}\left(x_{i}\right) \leq a^{L}\left(x_{j}\right)$, then $\partial^{* a}{ }_{j i}=1$.

If it is the other case, then $\partial_{j i}^{* a}=*$.

$\partial^{* a}{ }_{j i}$ is the possibility that the object $x_{j}$ is superior to the object $x_{i}$ under the attribute $a$.

The properties obtained by definition 2 are following:

(1) $\partial^{* a}=0.5$;

(2) $\partial^{* a} \in[0,1] \Leftrightarrow \partial^{* a}{ }_{j i}^{*} \neq$;

(3) $\partial_{i j}^{* a}=* \Leftrightarrow \partial^{*} a i=*$;

(4) $\partial_{j i}^{* a}+\partial_{i j}^{* a}=1 \Leftrightarrow \partial_{j i}^{* a} \neq * \vee \partial_{i j}^{* a} \neq *$.

\subsection{Range of Extended Possibility Degree $\partial^{*}$}

In the complete interval-value information system, if extended possibility that the object $x_{j}$ is superior to the object $x_{i}$ under the attribute $a$ is $\partial_{j i}^{* a}$, then extended possibility that the object $x_{j}$ is inferior to the object $x_{i}$ under the attribute $a$ is $\partial_{i j}^{* a}$. 
If the object $x_{j}$ is superior to the object $x_{i}$ under the attribute $a$ in the true sense, then $\partial_{j i}^{* a} \geq \partial_{i j}^{* a}$. Since, $\partial_{j i}^{* a}+\partial_{i j}^{* a}=1$ and $\partial_{j i}^{* a} \in[0,1], 0.5 \leq \partial_{j i}^{* a} \leq 1$ is obtained.

In the incomplete interval-valued information system, if $\partial^{*} a$ the object $x_{j}$ is superior to the object $x_{i}$ under the attribute $a$.

In summary, in order to ensure the reasonable classification, the range of extended possibility degree should be $\partial^{*} \in[0.5,1] \vee \partial^{*}=*$.

\subsection{Extended possibility degree dominance relation}

Definition 3: Given an incomplete interval-valued information systems $S=(U, A, V, f)$, the subset of the attributes $B(B \subseteq A)$, extended possibility degree dominance relation $R_{B}^{\geq}$and its class of advantage $\left[x_{i}\right]_{B}^{\geq}$about $B$ are defined as:

$$
\begin{gathered}
R_{B}^{2}=\left\{\left(x_{j}, x_{i}\right) \in U \times U \mid\left(\partial^{* a}{ }_{j i} \geq 0.5\right) \vee\left(\partial_{j i}^{* a}=*\right), \forall a \in B\right\} \\
{\left[x_{i}\right]_{B}^{\geq}=\left\{x_{j} \in U \mid\left(x_{j}, x_{i}\right) \in \mathrm{R}_{B}^{\geq}\right\}}
\end{gathered}
$$

The properties obtained by definition 3 are following:

(1) $R_{B}^{\geq}=\bigcap_{a \in B} R_{\{a\}}^{\geq}$;

(2) If $C \subseteq B \subseteq A$, then $R_{A}^{2} \subseteq R_{B}^{2} \subseteq R_{C}^{2}$;

(3) If $C \subseteq B \subseteq A$, then $\left[x_{i}\right]_{A}^{2} \subseteq\left[x_{i}\right]_{B}^{2} \subseteq\left[x_{i}\right]_{C}^{2}$;

(4) $R_{B}^{\geq}$meets the reflexivity, but it does not meet symmetry and transitivity.

\section{Improved Ranking Method}

According to the definition of classical dominance relation, all attributes of an object must be superior to ones of another object. But in real life, when objects have too many attributes that have advantages and disadvantages among them, it is so difficult to meet the definition of classical dominance relation that the ranking may fail. Obviously, the reasonable ranking result cannot be obtained. For example, between one object $x=\{2,8,4,7,2,1,0,9\}$ and object $y=\{3,4,5,1,0,0,1,2\}$, other attributes of the object $x$ are superior to the corresponding ones of the object $y$ except for the first, the third and the seventh attributes. According to the definition of classical dominance relation, the object $x$ is neither superior nor inferior to the object $y$. At this time, it will lead to the ranking failure. In fact, if at least four attributes of the object $x$ are superior to the corresponding ones of the object $y$, we will think that the object $x$ is superior to the object $y$.

If the objects $x$ and $y$ have both $m$ attributes, the number of attributes that the object $x$ is superior to the object $y$ is $N$, the number of attributes that the object $x$ is inferior to the object $y$ is $M$, then $N+M=m$. If the object $x$ is superior to the object $y$ in the real sense, then $N>M$. Hence, $N>\frac{m}{2}$. Because an object has integer attributes, the range of $N$ should be $[m / 2+1, m]$, where, / means to get the integer.

Based on the above analysis, when the object $x$ is superior to the object $y$, $N \in[m / 2+1, m]$ is reasonable.

Different extended possibility degree dominance relations and their classes of advantage are obtained because of different $N$. 
Definition 4: Given an incomplete interval-valued information systems $S=(U, A, V, f)$, the subset of the attributes $B(B \subseteq A)$, the number of comparable attributes is $N$, extended possibility degree dominance relation $R_{B_{N}}^{2}$ and its class of advantage $\left[x_{i}\right]_{B_{N}}^{2}$ are defined as:

$$
\begin{gathered}
R_{B_{N}}^{\geq}=\left\{\left(x_{j}, x_{i}\right) \in U \times U \mid\left(\partial^{* a}{ }_{j i} \geq 0.5\right) \vee\left(\partial^{* a}{ }_{j i}=*\right), \forall a \in B\right\} \\
{\left[x_{i}\right]_{B_{N}}^{\geq}=\left\{x_{j} \in U \mid\left(x_{j}, x_{i}\right) \in \mathrm{R}_{B_{N}}^{\geq}\right\}}
\end{gathered}
$$

Property 1 Given $B \subseteq A$, then

(1) if $N_{1} \leq N_{2}$, then $\left|\left[x_{i}\right]_{B_{N_{1}}}^{2}\right| \geq\left|\left[x_{i}\right]_{B_{N 2}}^{2}\right|$;

(2) if $B_{N_{1}} \subseteq B_{N_{2}}$, then $R_{B_{N_{1}}}^{\geq} \supseteq R_{B_{N_{2}}}^{2}$;

(3) if $B_{N_{1}} \subseteq B_{N_{2}}$, then $\left[x_{i}\right]_{B_{N_{1}}}^{\geq} \supseteq\left[x_{i}\right]_{B_{N 2}}^{\geq}$.

Proof: (1) The smaller $N$ is, the more objects that meet extended possibility degree $\partial^{*} \in[0.5,1]$ are. Therefore, $\left|\left[x_{i}\right]_{B_{N_{1}}}^{2}\right| \geq\left|\left[x_{i}\right]_{B_{N 2}}^{2}\right|$.

(2) If $B_{N_{1}} \subseteq B_{N_{2}}$, then $N_{1} \leq N_{2}$. The smaller $N$ is, the more objects that meet extended possibility degree $\partial^{*} \in[0.5,1]$ are. Therefore, objects in the extended possibility degree dominance relation are more. Namely, $R_{B_{N_{1}}}^{\geq} \supseteq R_{B_{N_{2}}}^{\geq}$.

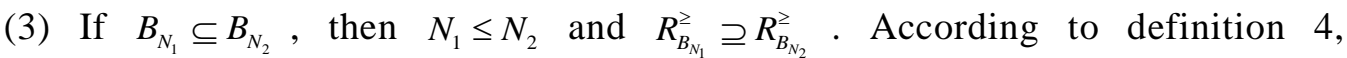
$\left[x_{i}\right]_{B_{N_{1}}}^{2} \supseteq\left[x_{i}\right]_{B_{N_{2}}}^{2}$ can be proved.

Definition 5: Given an incomplete interval-valued information systems $S=(U, A, V, f)$, the subset of the attributes $B(B \subseteq A)$, dominance degree of objects $x_{i}, x_{j}$ about $R_{B_{N}}^{\geq}$is defined as:

$$
R_{B_{N}}\left(x_{i}, x_{j}\right)= \begin{cases}\frac{\left|\left[x_{j}\right]_{B_{N}}^{2}-\left[x_{i}\right]_{B_{N}}^{2}\right|}{\left|\left[x_{j}\right]_{B_{N}}^{2}-\left[x_{i}\right]_{B_{N}}^{2}\right|+\left|\left[x_{i}\right]_{B_{N}}^{2}-\left[x_{j}\right]_{B_{N}}^{2}\right|}, & {\left[x_{j}\right]_{B_{N}}^{2} \neq\left[x_{i}\right]_{B_{N}}^{2}} \\ 0.5 & , \quad\left[x_{j}\right]_{B_{N}}^{2}=\left[x_{i}\right]_{B_{N}}^{2}\end{cases}
$$

Where $N \in[m / 2+1, m],\left[x_{j}\right]_{B_{N}}^{2}-\left[x_{i}\right]_{B_{N}}^{2}$ represents the parts that object $x_{i}$ is superior to object $x_{j}$. In like manner, $\left[x_{i}\right]_{B_{N}}^{2}-\left[x_{j}\right]_{B_{N}}^{2}$ represents the parts that object $x_{j}$ is superior to object $x_{i} \cdot|\cdot|$ represents the cardinality of a set.

The comprehensive dominance degree of object $x_{i}$ about $R_{B_{N}}^{\geq}$is defined as:

$$
R_{B_{N}}\left(x_{i}\right)=\frac{1}{n} \sum_{j=1}^{n} R_{B_{N}}\left(x_{i}, x_{j}\right)
$$

Property 2 Given $x_{i} \in U, n=U, B \subseteq A$, then

(1) $R_{B_{N}}\left(x_{i}, x_{j}\right) \in[0,1]$;

(2) $R_{B_{N}}\left(x_{i}, x_{i}\right)=0.5$

(3) $R_{B_{N}}\left(x_{i}, x_{j}\right)+R_{B_{N}}\left(x_{j}, x_{i}\right)=1$;

(4) $\sum_{i=1}^{n} \sum_{j=1}^{n} R_{B_{N}}\left(x_{i}, x_{j}\right)=\frac{n^{2}}{2}$;

(5) $R_{B_{N}}\left(x_{i}\right) \in\left[\frac{1}{2 n}, 1-\frac{1}{2 n}\right]$;

(6) $\sum_{i=1}^{n} R_{B_{N}}\left(x_{i}\right)=\frac{n}{2}$. 
Proof: (1) If $\left[x_{j}\right]_{B_{N}}^{2} \subseteq\left[x_{i}\right]_{B_{N}}^{2}$, then $\left|\left[x_{j}\right]_{B_{N}}^{2}-\left[x_{i}\right]_{B_{N}}^{2}\right|=0$. Thus $R_{B_{N}}\left(x_{i}, x_{j}\right)=0$. At this time, $R_{B_{N}}\left(x_{i}, x_{j}\right)$ gets the minimum value.

If $\left[x_{j}\right]_{B_{N}}^{2} \supseteq\left[x_{i}\right]_{B_{N}}^{2}$, then $R_{B_{N}}\left(x_{i}, x_{j}\right)=1$. At this time, $R_{B_{N}}\left(x_{i}, x_{j}\right)$ gets the maximum value.

In summary, $R_{B_{N}}\left(x_{i}, x_{j}\right) \in[0,1]$ is proved.

(2) According to definition 5, if $\left[x_{j}\right]_{B_{N}}^{2}=\left[x_{i}\right]_{B_{N}}^{2}$, then $R_{B_{N}}\left(x_{i}, x_{j}\right)=0.5$. Since $\left[x_{i}\right]_{B_{N}}^{2}=\left[x_{i}\right]_{B_{N}}^{2}$, then $R_{B_{N}}\left(x_{i}, x_{i}\right)=0.5$.

(3) According to definition 5,

if $\left[x_{j}\right]_{B_{N}}^{2}=\left[x_{i}\right]_{B_{N}}^{2}$, then $R_{B_{N}}\left(x_{i}, x_{j}\right)=0.5, R_{B_{N}}\left(x_{j}, x_{i}\right)=0.5$. Obviously, $R_{B_{N}}\left(x_{i}, x_{j}\right)+R_{B_{N}}\left(x_{j}, x_{i}\right)=1$.

if $\left[x_{j}\right]_{B_{V}}^{2} \neq\left[x_{i}\right]_{B_{N}}^{2}$, then

$$
\begin{aligned}
& R_{B_{N}}\left(x_{i}, x_{j}\right)=\frac{\left|\left[x_{j}\right]_{B_{N}}^{2}-\left[x_{i}\right]_{B_{N}}^{2}\right|}{\left|\left[x_{j}\right]_{B_{N}}^{2}-\left[x_{i}\right]_{B_{N}}^{2}\right|+\left|\left[x_{i}\right]_{B_{N}}^{2}-\left[x_{j}\right]_{B_{N}}^{2}\right|}, \\
& R_{B_{N}}\left(x_{j}, x_{i}\right)=\frac{\left|\left[x_{i}\right]_{B_{N}}^{2}-\left[x_{j}\right]_{B_{N}}^{2}\right|}{\left|\left[x_{i}\right]_{B_{N}}^{2}-\left[x_{j}\right]_{B_{N}}^{2}\right|+\left|\left[x_{j}\right]_{B_{N}}^{2}-\left[x_{i}\right]_{B_{N}}^{2}\right|} .
\end{aligned}
$$

Hence,

$$
R_{B_{N}}\left(x_{i}, x_{j}\right)+R_{B_{N}}\left(x_{j}, x_{i}\right)=\frac{\left|\left[x_{j}\right]_{B_{N}}^{2}-\left[x_{i}\right]_{B_{N}}^{2}\right|}{\left|\left[x_{j}\right]_{B_{N}}^{2}-\left[x_{i}\right]_{B_{N}}^{2}\right|+\left|\left[x_{i}\right]_{B_{N}}^{2}-\left[x_{j}\right]_{B_{N}}^{2}\right|}+\frac{\left|\left[x_{i}\right]_{B_{N}}^{2}-\left[x_{j}\right]_{B_{N}}^{2}\right|}{\left|\left[x_{i}\right]_{B_{N}}^{2}-\left[x_{j}\right]_{B_{N}}^{2}\right|+\left|\left[x_{j}\right]_{B_{N}}^{2}-\left[x_{i}\right]_{B_{N}}^{2}\right|}=1 .
$$

In summary, $R_{B_{N}}\left(x_{i}, x_{j}\right)+R_{B_{N}}\left(x_{j}, x_{i}\right)=1$ is proved.

(4) According to $R_{B_{N}}\left(x_{i}, x_{i}\right)=0.5$ and $R_{B_{N}}\left(x_{i}, x_{j}\right)+R_{B_{N}}\left(x_{j}, x_{i}\right)=1$,

$$
\sum_{i=1}^{n} \sum_{j=1}^{n} R_{B_{N}}\left(x_{i}, x_{j}\right)=0.5 \times n+1+2+\cdots+n-1=\frac{n^{2}}{2} \text {. }
$$

(5) The worst case is that $R_{B_{N}}\left(x_{i}, x_{j}\right)=0$ except for $R_{B_{N}}\left(x_{i}, x_{i}\right)=0.5$, therefore, $R_{B_{N}}\left(x_{i}\right)=\frac{0+\cdots+0.5+\cdots+0}{n}=\frac{1}{2 n}$.

The best case is that $R_{B_{N}}\left(x_{i}, x_{j}\right)=1$ except for $R_{B_{N}}\left(x_{i}, x_{i}\right)=0.5$, therefore, $R_{B_{N}}\left(x_{i}\right)=\frac{1+\cdots+0.5+\cdots+1}{n}=\frac{n-1+0.5}{n}=1-\frac{1}{2 n}$.

In summary, $R_{B_{N}}\left(x_{i}\right) \in\left[\frac{1}{2 n}, 1-\frac{1}{2 n}\right]$ is proved.

(6)

$$
\begin{aligned}
\sum_{i=1}^{n} R_{B_{N}}\left(x_{i}\right)= & \sum_{i=1}^{n} \frac{1}{n} \sum_{j=1}^{n} R_{B_{N}}\left(x_{i}, x_{j}\right) \\
& =\frac{1}{n} \sum_{i=1}^{n} \sum_{j=1}^{n} R_{B_{N}}\left(x_{i}, x_{j}\right) \\
& =\frac{1}{n} \times \frac{n^{2}}{2} \\
& =\frac{n}{2}
\end{aligned}
$$

Different comprehensive dominance degrees are obtained because of different $N$. Thus, Average comprehensive dominance degree is introduced and used to rank for all objects. It is the average value of all comprehensive dominance. 
Definition 6: Given an incomplete interval-valued information systems $S=(U, A, V, f)$, the subset of the attributes $B(B \subseteq A)$, Average comprehensive dominance degree of objects $x_{i}$ about $R_{B_{N}}^{2}$ is defined as:

$$
R_{B}\left(x_{i}\right)=\frac{1}{(m-1) / 2+1} \sum_{N=m / 2+1}^{m} R_{B_{N}}\left(x_{i}\right)
$$

Average comprehensive dominance degree $R_{B}\left(x_{i}\right)$ represents the degree that object $x_{i}$ is superior to another object, and then the greater $R_{B}\left(x_{i}\right)$ is, the better object $x_{i}$ is.

Property 3 Given $x_{i} \in U, n=U, B \subseteq A$, then

(1) $R_{B}\left(x_{i}\right) \in\left[\frac{1}{2 n}, 1-\frac{1}{2 n}\right]$

(2) $\sum_{i=1}^{n} R_{B}\left(x_{i}\right)=\frac{n}{2}$

Proof: (1) According to $R_{B_{N}}\left(x_{i}\right) \in\left[\frac{1}{2 n}, 1-\frac{1}{2 n}\right]$,

if $R_{B_{N}}\left(x_{i}\right)=\frac{1}{2 n}$ for any $N$ value,

$$
\begin{aligned}
R_{B}\left(x_{i}\right)_{\min } & =\frac{1}{(m-1) / 2+1} \sum_{N=m / 2+1}^{m} R_{B_{N}}\left(x_{i}\right) \\
& =\frac{1}{(m-1) / 2+1} \times((m-1) / 2+1) \times \frac{1}{2 n} \\
& =\frac{1}{2 n}
\end{aligned}
$$

if $R_{B_{N}}\left(x_{i}\right)=1-\frac{1}{2 n}$ for any $N$ value,

$$
\begin{aligned}
R_{B}\left(x_{i}\right)_{\max } & =\frac{1}{(m-1) / 2+1} \sum_{N=m / 2+1}^{m} R_{B_{N}}\left(x_{i}\right) \\
& =\frac{1}{(m-1) / 2+1} \times((m-1) / 2+1) \times\left(1-\frac{1}{2 n}\right) \\
& =1-\frac{1}{2 n}
\end{aligned}
$$

In summary, $R_{B}\left(x_{i}\right) \in\left[\frac{1}{2 n}, 1-\frac{1}{2 n}\right]$ is proved.

(2) According to $\sum_{i=1}^{n} R_{B_{N}}\left(x_{i}\right)=\frac{n}{2}$,

$$
\begin{aligned}
\sum_{i=1}^{n} R_{B}\left(x_{i}\right) & =\sum_{i=1}^{n} \frac{1}{(m-1) / 2+1} \sum_{N=m / 2+1}^{m} R_{B_{N}}\left(x_{i}\right) \\
= & \frac{1}{(m-1) / 2+1} \sum_{i=1}^{n} \sum_{N=m / 2+1}^{m} R_{B_{N}}\left(x_{i}\right) \\
= & \frac{1}{(m-1) / 2+1} \sum_{N=m / 2+1}^{m} \sum_{i=1}^{n} R_{B_{N}}\left(x_{i}\right) \\
= & \frac{1}{(m-1) / 2+1} \times((\mathrm{m}-1) / 2+1) \times \frac{n}{2} \\
= & \frac{n}{2}
\end{aligned}
$$


Therefore, $\sum_{i=1}^{n} R_{B}\left(x_{i}\right)=\frac{n}{2}$ is proved.

The concrete steps of ranking method based on extended possibility degree dominance relation are following:

Step 1: According to incomplete interval-valued information, all extended possibility degrees $\partial^{*}$ are obtained;

Step 2: According to the number of the attributes, the range of $N$ is confirmed;

Step 3: According to different $N$ values, extended possibility degree dominance relations and classes of advantage are obtained, and then the corresponding dominance matrixes are constructed;

Step 4: According to different $N$ values, the corresponding comprehensive dominance degrees $R_{B_{Y}}\left(x_{i}\right)$ are obtained ;

Step 5: According to $R_{B_{V}}\left(x_{i}\right)$, average comprehensive dominance degrees of all objects $R_{B}\left(x_{i}\right)$ are obtained;

Step 6: According to $R_{B}\left(x_{i}\right)$, the ranking result can be obtained.

\section{Example Analysis}

Table 1 gives an incomplete interval-valued information system, where $U=\left\{x_{1}, x_{2} \cdot x_{3}, x_{4}, x_{5}, x_{6}, x_{7}\right\}, A=\left\{a_{1}, a_{2}, a_{3}, a_{4}, a_{5}\right\}$.

Table 1. Incomplete Interval-Valued Information System

\begin{tabular}{cccccc}
\hline & $a_{1}$ & $a_{2}$ & $a_{3}$ & $a_{4}$ & $a_{5}$ \\
\hline$x_{1}$ & {$[2.17,2,86]$} & {$[2.45, *]$} & {$[5.32,7.23]$} & {$[3.21,3.95]$} & {$[2.54,3.12]$} \\
$x_{2}$ & {$[1.35,2.12]$} & {$[1.42,2.09]$} & {$[*, 3.93]$} & {$[1.87,2.62]$} & {$[1.67,2.32]$} \\
$x_{3}$ & {$[*, *]$} & {$[3.37,5.11]$} & {$[6.37,10.28]$} & {$[3.76,5.70]$} & {$[*, 5.28]$} \\
$x_{4}$ & {$[2.29,3.43]$} & {$[2.60, *]$} & {$[6.71,8.81]$} & {$\left[{ }^{*}, *\right]$} & {$[3.01,3.84]$} \\
$x_{5}$ & {$[2.22,3.07]$} & {$[2.43,3,32]$} & {$[4.37, *]$} & {$[2.66,3.68]$} & {$[2.39,3.20]$} \\
$x_{6}$ & {$[2.51,4.04]$} & {$[2.52,4.12]$} & {$[7.12,11.26]$} & {$[4.44,6.91]$} & {$[3.06,4.65]$} \\
$x_{7}$ & {$[1.24, *]$} & {$[1.35,1.91]$} & {$[3.83,4.28]$} & {$[2.13,3.01]$} & {$[1.72,2.34]$} \\
\hline
\end{tabular}

According to the data in the Table 1, the concrete steps of ranking method based on extended possibility degree dominance relation are following:

Step 1: According to incomplete interval-valued information, all extended possibility degrees $\partial^{*}$ are obtained;

Step 2: According to the number of the attributes, the range of $N$ is $[3,5]$;

Step 3: When $N=3$, extended possibility degree classes of advantage of all objects are following:

$$
\begin{gathered}
{\left[x_{1}\right]_{B_{3}}^{2}=\left\{x_{1}, x_{3}, x_{4}, x_{5}, x_{6}\right\},\left[x_{2}\right]_{B_{3}}^{2}=\left\{x_{1}, x_{2}, x_{3}, x_{4}, x_{5}, x_{6}, x_{7}\right\},\left[x_{3}\right]_{B_{3}}^{2}=\left\{x_{1}, x_{3}, x_{4}, x_{5}, x_{6}\right\},} \\
{\left[x_{4}\right]_{B_{3}}^{2}=\left\{x_{3}, x_{4}, x_{5}, x_{6}\right\},\left[x_{5}\right]_{B_{3}}^{2}=\left\{x_{1}, x_{3}, x_{4}, x_{5}, x_{6}\right\},\left[x_{6}\right]_{B_{3}}^{2}=\left\{x_{3}, x_{6}\right\},} \\
{\left[x_{7}\right]_{B_{3}}^{2}=\left\{x_{1}, x_{2}, x_{3}, x_{4}, x_{5}, x_{6}, x_{7}\right\}}
\end{gathered}
$$

According to extended possibility degree classes of advantage, the dominance matrix is constructed: 


$$
R_{B_{3}}\left(x_{i}, x_{j}\right)=\left[\begin{array}{ccccccc}
\frac{1}{2} & 1 & \frac{1}{2} & 0 & \frac{1}{2} & 0 & 1 \\
0 & \frac{1}{2} & 0 & 0 & 0 & 0 & \frac{1}{2} \\
\frac{1}{2} & 1 & \frac{1}{2} & 0 & \frac{1}{2} & 0 & 1 \\
1 & 1 & 1 & \frac{1}{2} & 1 & 0 & 1 \\
\frac{1}{2} & 1 & \frac{1}{2} & 0 & \frac{1}{2} & 0 & 1 \\
1 & 1 & 1 & 1 & 1 & \frac{1}{2} & 1 \\
0 & \frac{1}{2} & 0 & 0 & 0 & 0 & \frac{1}{2}
\end{array}\right]
$$

Step 4: When $N=3$, the corresponding comprehensive dominance degrees of all objects are following:

$$
R_{B_{3}}\left(x_{1}\right)=\frac{1}{2}, R_{B_{3}}\left(x_{2}\right)=\frac{1}{7}, R_{B_{3}}\left(x_{3}\right)=\frac{1}{2}, R_{B_{3}}\left(x_{4}\right)=\frac{11}{14}, R_{B_{3}}\left(x_{5}\right)=\frac{1}{2}, R_{B_{3}}\left(x_{6}\right)=\frac{13}{14}, R_{B_{3}}\left(x_{7}\right)=\frac{1}{7}
$$

In like manner, when $N=4$ and $N=5$, the corresponding comprehensive dominance degrees of all objects are following respectively:

$$
\begin{aligned}
& R_{B_{4}}\left(x_{1}\right)=\frac{1}{2}, R_{B_{4}}\left(x_{2}\right)=\frac{1}{14}, R_{B_{4}}\left(x_{3}\right)=\frac{5}{7}, R_{B_{4}}\left(x_{4}\right)=\frac{5}{7}, R_{B_{4}}\left(x_{5}\right)=\frac{5}{14}, R_{B_{4}}\left(x_{6}\right)=\frac{13}{14}, R_{B_{4}}\left(x_{7}\right)=\frac{3}{14} ; \\
& R_{B_{5}}\left(x_{1}\right)=\frac{3}{7}, R_{B_{5}}\left(x_{2}\right)=\frac{1}{7}, R_{B_{5}}\left(x_{3}\right)=\frac{6}{7}, R_{B_{5}}\left(x_{4}\right)=\frac{9}{14}, R_{B_{5}}\left(x_{5}\right)=\frac{3}{7}, R_{B_{5}}\left(x_{6}\right)=\frac{6}{7}, R_{B_{5}}\left(x_{7}\right)=\frac{1}{7}
\end{aligned}
$$

Step 5: Average comprehensive dominance degrees of all objects are following:

$$
\begin{aligned}
R_{B}\left(x_{1}\right)=0.476, R_{B}\left(x_{2}\right)= & 0.119, R_{B}\left(x_{3}\right)=0.690, R_{B}\left(x_{4}\right)=0.714, R_{B}\left(x_{5}\right)=0.429, \\
& R_{B}\left(x_{6}\right)=0.905, R_{B}\left(x_{7}\right)=0.167 .
\end{aligned}
$$

Step 6: The ranking result is following:

$$
x_{6} \succ x_{4} \succ x_{3} \succ x_{1} \succ x_{5} \succ x_{7} \succ x_{2}
$$

Comparing with other ranking methods in different examples, the ranking results are following:

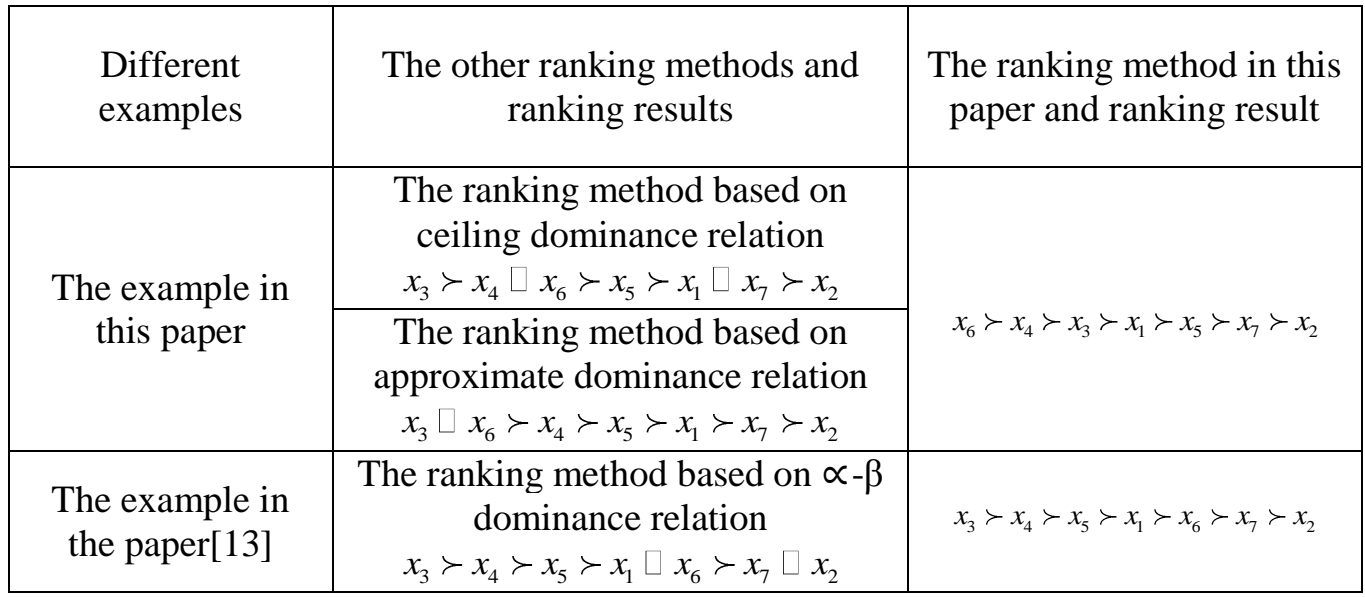

Analysis of the ranking results :

Judging from the ranking results, the ranking method in this paper can distinguish the objects better. However, the other three ranking methods cannot distinguish them better. Therefore, the ranking method in this paper has stronger differentiation degree. 
Judging from the ranking methods, the definitions of ceiling dominance relation and approximate dominance relation are too loose. Moreover, the corresponding ranking methods may lead to the ranking failure. The ranking method based on $\alpha-\beta$ dominance relation has such strong subjectivity that it makes the ranking result unreasonable. However, the ranking method in this paper can improve the deficiencies of above ranking methods. Moreover, it can avoid the subjectivity to make the ranking result more objective and reasonable. Besides, it can solve the problem that classical dominance relation leads to ranking failure.

\section{Conclusions}

In order to solve the problem that too many attributes maybe result in the ranking failure in incomplete interval-valued information systems, a new ranking method based on extended possibility degree dominance relation is proposed. An example verifies that this new ranking method is feasible. Comparing with the other ranking methods, this new ranking method has not only simpler operation and smaller calculation, but also can solve the problem that classical dominance relation may lead to ranking failure. Moreover, it can not only avoid the subjectivity, but also make the ranking result more objective and reasonable. Besides, it can not only have stronger differentiation degree, but also have wide application.

This paper focuses on the ranking problem in the incomplete interval-valued information systems. Attribute reduction in the incomplete interval-valued information systems will be the next important research.

\section{Acknowledgements}

This research was supported by the National Natural Science Funds of China under Grant No. 61263047; the Nature Science Foundation of Gansu Province under Grant No. 2011GS04147. These supports are gratefully acknowledged.

\section{References}

[1] Z. Pawlak, "Rough Sets", International Journal of Computer and Information Sciences, vol.11,no.5, (1982), pp.341-356.

[2] S. Greco, B. Matarazzo and R. Slowinski, "A New Rough Set Approach to Multicriteria and Multiattribute Classification”, Rough Sets and Current Trends in Computing, (1998), pp.60-67.

[3] S. Greco, B. Matarazzo and R. Slowinski, "Rough Sets Theory for Multicriteria Decision Analysis", European Journal of Operational Research, vol.129, no.1, (2001), pp.1-47.

[4] S. Greco, B.o Matarazzo and R. Slowinski, "Rough Sets Methodology for Sorting Problems in Presence of Multiple Attributes and Criteria”, European Journal of Operational Research, vol.138, no.2, (2002), pp.247-259.

[5] J. Liu, L. Xue and S. F. Liu, "Research on Multiple-attribute Decision Making Problems Based on the Superiority index", Control and Decision, vol.25, no.7, (2010), pp.1079-1087.

[6] J. Liu, S. F. Liu and S. X. Wu, "Ranking Research Based on Dominant Relation for Multiple-attribute", Control and Decision, vol.27, no.4, (2012), pp.632-640.

[7] W. C. Chen, Y. J. Lv and S. Z Weng, "Sorting Method and its Application Based on Tolerance Dominance Relation”, Journal of Computer Applications, vol.34, no.8, (2014), pp.2170-2174.

[8] L. D. Wang, X. J. Tian and Y. B. Yang, "The Comprehensive Evaluation of Teaching Based on Entropy Weight and Dominance Relation", Journal of Mathematics in Practice and Theory, vol.44, no.10, (2014), pp.9-12.

[9] J. P. Li, C. Y. Yue and W. Li, "A Dominance Relation-based Decision Making Approach for Multiattribute Decision Making Problems with Incomplete Information", Control and Decision, vol.28, no.2, (2013), pp.229-234.

[10] L. Zhao, X. Zhang and Z. Xue, "Security Assessment for Incomplete Interval-valued Information System", Computer Engineering, vol.37, no.11, (2011), pp.147-148.

[11] D. S. Qiu, C. He and X. M. Zhu, "Ranking Method Research of Interval Numbers Based on Probability Reliability Distribution", Control and Decision, vol.27, no.12, (2012), pp.1895-1898. 
[12] Q. S. Yang, G. Y. Wang and Q. H. Zhang, "The Interval-valued Rough Set Extended Model Based on the Dominance Relation", Journal of Shandong University (Natural Science), vol.45, no.9, (2010), pp.713.

[13] M. L. Hu and L. L. Li, "A Novel Dominance Relation and Application in Interval Grey Number Decision Model”, Journal of Grey System, vol.26, no.1, (2014), pp.91-98.

[14] B. Wang, M. W. Shao and J. H. Wang, "New Evaluation Model for Incomplete Interval-valued Information System Based on Improved Dominance Relations”, Computer Science, vol.41, no.2, (2014), pp.253-256.

[15] L. J. Yang, “A few Kinds of Incomplete Information Systems' Attribute Reduction”, M. S. Thesis , School of Mathematics and Software Science, Sichuan Normal University, (2014).

\section{Authors}

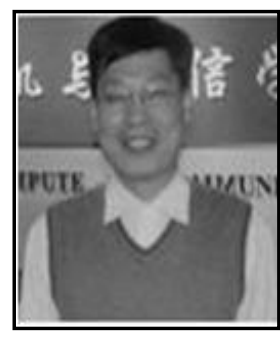

Qiwen Zhang, he was born in 1975. He is an associate professor and masters' tutor of Lanzhou University of Technology. The main research fields are data dimming, pattern recognition, image processing and knowledge engineering and so on.

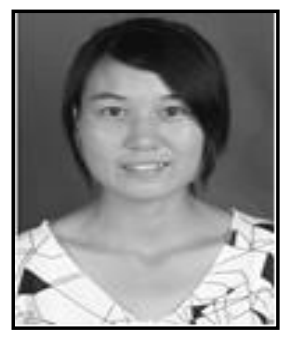

Xueqin Wang, she was born in 1989. She is a master student of Lanzhou University of Technology. The main research fields are artificial intelligence and data mining. 
International Journal of Hybrid Information Technology Vol. 9, No.6 (2016) 